\title{
Relaciones entre la Violencia, Salud Mental, Participación Ciudadana, Y Actitudes hacia la Justicia Transicional en la Costa Caribe de Colombia ${ }^{1}$
}

\author{
Relationships found between Political Violence, Mental Health, Participation \\ of Citizens, and Attitudes toward Transitional Justice in the Caribbean Coast of \\ CoLombia.
}

Por: Laura K. Taylor.

Ph.D in Psychology and Peace Studies. Filiación institucional: Departmen of Psichology and Kroe Institute for International Peace Studies, University Of Notre Dame. E-mail: ltaylo12@nd.edu

Artículo de investigación científica y tecnológica según clasificación Colciencias.

${ }^{1}$ Esta investigación se realizó con el aporte del Kellogg Institute for Internationa Studies, Kroc Institute for International Peace Studies, y Institute for Scholarship y Institute for Scholarship in the Liberal Arts de University of Notre Dame. Agradezco especialmente a los participantes en el pilotaje de las diferentes comunidades en la costa Caribe; los equipos de investigación de la Universidad del Sinú, Córdoba , $Y$ del CECAR, Córdoba, y del CECAR, Sucre, la Comisión Ciudadana para Reconciliación en el Caribe (CCRC); y John
Paul Lederach, María Lucía Zapata, y los aprendices.
Articulo recibido: Julio 25 de 2011. Artículo aprobado: Noviembre 17 de 2011

\section{RESUMEN}

El presente artículo explora por un lado que la violencia política tiene el potencial de amenazar el bienestar de los individuos y romper el tejido social, pero por otro lado, que los individuos y comunidades son resilientes frente a ella. De este modo, planteamos la siguiente pregunta de investigación: ¿Cómo las experiencias de violencia política afectan a las personas a nivel personal? Específicamente este artículo explorará las relaciones entre la violencia, salud mental, participación ciudadana, y actitudes hacia la justicia transicional y democracia dentro un contexto de conflicto armado prolongado. Una contribución teórica de este proyecto es la integración de dos marcos teóricos de psicología y estudios de paz. En el pilotaje se realizaron 184 encuestas con individuos, los cuales revelan determinados patrones. Por ejemplo, que diferentes tipos de violencia están relacionados con diferentes problemas de salud mental y maneras de enfrentar situaciones difíciles. El artículo también revela que a pesar del apoyo ciudadano hacia la democracia y la justicia transicional, muchos colombianos no están satisfechos con las iniciativas actuales que se adelantan desde el nivel nacional. Los resultados sugieren la necesidad de desarrollar intervenciones de salud mental y construcción de paz que son más sensibles al contexto y menos centralizadas.

Palabras claves: Violencia política, salud mental, resiliencia (resilience), participación ciudadana, justicia transicional.

\section{ABSTRACT}

The current article investigates how political violence can increase psychological stress and how it can tear down the social structure of a community. At the same time, we examine how individuals and communities are surprisingly resilient to the on-going conflict. This phenomenon leads us to this research question: How does the exposure to political violence affect people at an individual level? More precisely, this article explores what relationship we can find between political violence and individual mental health by examining the participation these individuals have in a community, and also by studying the attitudes or behaviors they had towards transitional justice and democracy, in a setting of a very long term armed conflict. A theoretical contribution of this project is the integration made between theoretical psychological frameworks and studies in favor of peace. Surveys of 184 individuals reveal certain trends, 


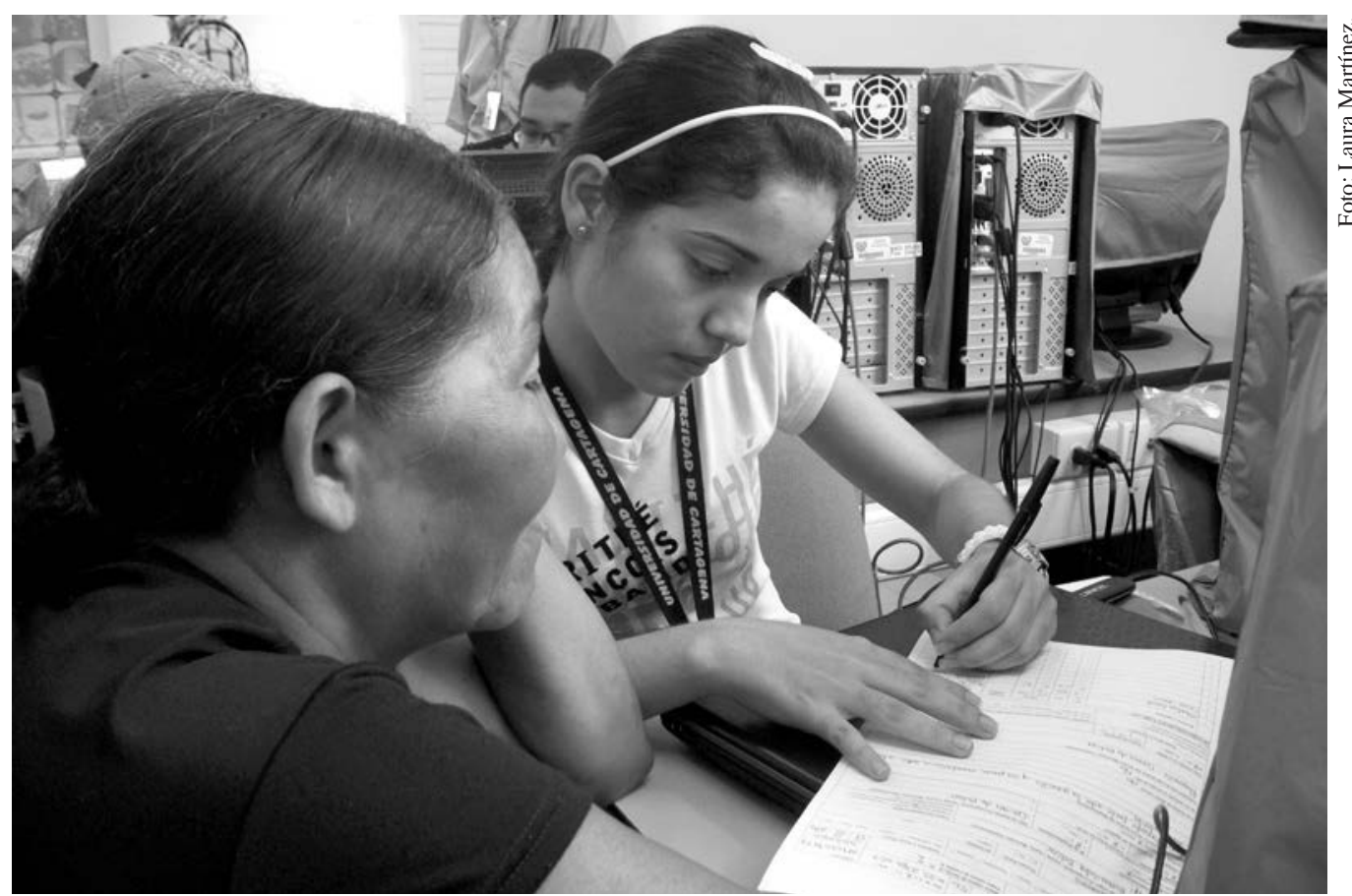

Archivo fotográfico del Observatorio de Cultura Política, Paz, Convivencia y Desarrollo en la subregión de los Montes de María. 2011

such as the one that shows that different types of violence are associated to different types of mental health and how these people solve problems. In addition, this article shows that despite the wide-spread actions taken to support democracy and transitional justice, in general, many Colombians are not satisfied with the current, national-level initiatives taken by the government. The results suggest that there is a need to work more deeply with people having mental health problems. The intervention that could be made in this aspect will show a decentralized process more sensitive and accurate to the process of peace the government is looking for in the contexts where these people live.

Key words: Political violence, mental health, resilience, civic participation, transitional justice.

\section{INTRODUCCIÓN}

Alrededor del mundo aumenta la atención al desarrollo y aplicación de mecanismos de justicia transicional en contextos de alta violencia, creados con el fin de reparar la herencia de violaciones sistemáticas de los derechos humanos (ICTJ, 2012). En particular, países todavía inmersos en conflictos prolongados utilizan diferentes métodos para apoyar a los ciudadanos. Muchos de ellos son dirigidos a sanar a las personas y poblaciones afectadas y construir una sociedad más estable, justa, e equitativa (Grodsky, 2009). Sin embargo, no existe suficiente información empírica sobre las actitudes y perspectivas de las personas más afectadas por la violencia ni de las políticas públicas de transformación de conflictos. En muchos países, incluyendo a Colombia, la mayoría de las políticas públicas no contempla las dimensiones emocionales y cognitivas 
de la población que se atiende en una manera suficiente (Shapiro, 2010). No obstante, si se quiere que los planes para resolver el conflicto a nivel nacional tengan un impacto real, debe haber cierto apoyo sicosocial en el nivel local (Lederach, 2005). Este estudio es un pilotaje dirigido a explorar las relaciones entre salud mental, o el bienestar de un individuo que se incluye la habilidad de afrontar estrés en una manera constructiva y cuidarse emocionalmente y físicamente, participación y conexiones comunales, y justicia transicional en una situación de conflicto prolongado.

\section{Marco teórico}

Una contribución original de este proyecto es la integración de la psicología y los estudios de paz como dos marcos teóricos que aunque distintos son complementarios: la ecología social del desarrollo humano (Bronfenbrenner, 1979) y del paradigma anidado para la transformación de conflicto (Lederarch, 1997; Figura 1). El enfoque del marco psicológico explica cuáles variables en el ámbito socio-cultural afectan los procesos emocionales y de comportamiento, sin embargo también llama la atención a la influencia mutua entre el individuo y su ecología social. El paradigma anidado complementa este marco psicológico; incluye los cambios personales y relacionales y a la misma vez se nota cómo estos cambios pueden sembrar e inspirar los procesos de justicia transicional a nivel nacional. Aunque los marcos tienen diferentes enfoques desarrollo humano y transformación de conflictos - los dos marcos incluyen influencias bidireccionales cruzando los diferentes niveles. La integración de estas teorías constituye la fundamentación teórica del pilotaje y este artículo.

Figura 1: (a) Ecología social del desarrollo humano (Bronfenbrenner, 1979) y (b) el paradigma anidado para la transformación de conflicto (Lederach, 1997; 2007)
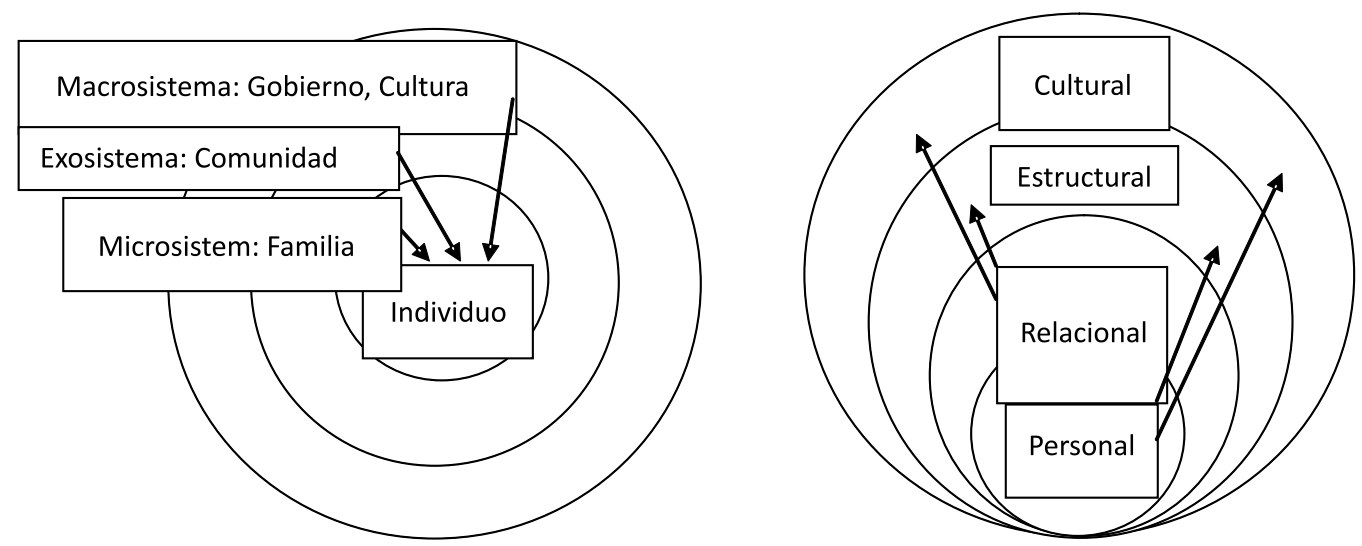


\section{Conflicto, sociedad civil, y justicia transicional en Colombia}

Colombia es un país afectado por más de 50 años de guerra civil y violencia, constituyéndose en un caso interesante para entender las relaciones entre bienestar y justicia transicional dentro un contexto de conflicto armado intenso por varias razones. Primero, la violencia no ocurre al mismo ritmo e intensidad por toda la geografía nacional. Aunque décadas de conflicto armado interno han afectado toda la geografía nacional, la costa Caribe tiene su propia historia. En las últimas dos décadas, el crecimiento del paramilitarismo, el narcotráfico, y el incremento de organizaciones criminales como consecuencia de la desmovilización de las Autodefensas Unidas de Colombia (AUC) resultado de la Ley de Justicia y Paz en 2005, han determinado las manifestaciones de violencia en esta región (Bouvier, 2009). Sin embargo, esta violencia no afecta a todas las personas de la misma manera. La literatura en psicología ha demostrado que la violencia amenaza el bienestar, aumentando el estrés psicológico, y puede romper el tejido social de la comunidad (Martín-Baró, 1996). Por otro lado, el sufrimiento también puede fomentar la cohesión social, apoyo mutuo, y sentido de empatía y solidaridad con otros (Staub \& Vollhart, 2008). Una meta de este artículo es entender cómo diferentes tipos (Ej., directa y indirecta) e intensidades (Ej., fatal o no fatal) de violencia afectan a las personas.

Segundo, en contraste con otros gobiernos en situaciones de guerra persistente, se categoriza Colombia como una democracia (Polity IV Country Report 2010: Colombia) participativa, un sistema que está organizado para resolver conflictos por medios no violentos. Sin embargo, los gobiernos democráticos no siempre tienen la capacidad para manejar conflictos efectivamente. Aparte de los aparatos formales del Estado que deberían equilibrar el poder y desacuerdos, Colombia tiene una sociedad civil fuerte (Bouvier, 2009), que es determinante para un sistema democrático. En otras partes del mundo, fuertes redes de la sociedad civil han prevenido que disturbios puntuales escalen hacia un conflicto prolongado (Varshney, 2001) y fortalecidas a procesos de reconciliación (Hamber, 2009). Otra meta de este artículo es describir las relaciones entra la violencia, salud mental, y la participación en la sociedad civil desde la perspectiva que esta participación puede servir como la 'levadura crítica,' en las palabras de Lederach (2005), para promover la transformación de conflicto.

Al final, el artículo de justicia transicional en medio de conflictos prolongados es una idea relativamente nueva, y Colombia es un caso importante para 
estudiar la complejidad de este fenómeno. En sitios de conflicto, actitudes hacia las iniciativas de justicia transicional, por ejemplo la búsqueda de la verdad, justicia, perdón, amnistía, son importantes para las relaciones interpersonales a nivel comunitario. Estas actitudes también están formadas por la confianza que un individuo tiene del gobierno actual y de su sistema democrático. En Colombia, la costa Caribe es un sitio importante no solo por los patrones de violencia y desmovilización, sino también porque la población en la región tiene diferentes percepciones y opiniones de justicia, verdad, y reconciliación (ICTJ, 2008). Por ejemplo, solo 67\% de encuestados en el Caribe manifestó su apoyo por un derecho individual a la verdad, en contraste con más de $80 \%$ en las otras cuatro regiones del país (ICTJ, 2008: 37). Del mismo modo, el porcentaje más alto de encuestados apoyó el derecho colectivo a la verdad, que se entendía como la construcción de un pasado compartido y la prevención a la recurrencia de la violencia (ICTJ, 2008: 39). Estos resultados identifican al Caribe como un área importante y enfatizan la necesidad para una investigación más profunda sobre las actitudes y opiniones de los individuos hacia diferentes mecanismos de justicia transicional.

Considerando la historia de Colombia, específicamente la costa Caribe, y la investigación psicológica previa sobre el impacto de la violencia política, y la variabilidad de las actitudes hacia la justicia transicional, este artículo se enfoca en una pregunta de investigación primaria, tomando en cuenta los niveles de la ecología social: ¿Cómo las experiencias violentas afectan las personas a nivel individual? Específicamente este artículo explorará el impacto de la violencia política en la salud mental del individuo, su participación en la sociedad civil, y sus actitudes hacia la justicia transicional dentro un contexto de conflicto prolongado.

\section{MÉTODO}

El pilotaje utilizó una combinación de métodos cuantitativos y cualitativos con el objetivo de tener un balance de información con datos que proporcionarán ángulos complementarios a la investigación y que incluyen información a nivel individual y colectivo. Este artículo presentará un resumen de los datos cuantitativos. Los beneficios de las encuestas son la habilidad de generalizar los resultados, recolectar información en una forma rápida y sistemática, y permitir la comprobación de hipótesis con una precisión aceptada por otras áreas de las ciencias sociales $(\mathrm{Ej} ., \mathrm{p}<.05)$. Un reto de este método es la limitación de explorar la profundidad de la información y de las experiencias 
complejas. El pilotaje fue aprobado por el Comité de Ética de la Universidad de Notre Dame.

Las encuestas fueron recolectadas durante agosto de 2010 por un equipo de quince investigadores de campo entre los que se encontraban profesores, y estudiantes de psicología o trabajo social de la Universidad del Sinú y el CECAR. Los investigadores participaron en una reunión para dirigirlos sobre el pilotaje y presentarles las metas y metodología del mismo. Igualmente se realizaron capacitaciones semanales para resolver las incertidumbres que se presentaban en el trabajo de campo.

\section{Áreas de investigación}

El pilotaje se realizó en 14 municipios de los departamentos de Córdoba y Sucre en sitios de investigación previamente seleccionados para capturar variabilidad específica en dos ejes: participación y violencia. Tomando en cuenta un balance entre comunidades urbanas y rurales, se eligió cuatro tipos de sitios con las siguientes combinaciones de perfiles: alta/baja violencia y alta/baja participación. El conocimiento regional de los equipos de las dos universidades locales vinculadas en la investigación fue determinante en el proceso de selección de los municipios, ya que incrementó la posibilidad de hacer comparaciones y nos permitió tener acceso a un perfil de participantes diverso.

\section{Participantes}

De los 184 participantes (49\% hombres) en la encuesta se encontró que el promedio de edad fue de 42.50 años $(S D=14.20)$ y 3.57 hijos $(S D=2.91)$. En cuanto al estado civil, un $77 \%$ de los participantes eran casados o en unión libre, $12 \%$ nunca han estado casados, $7 \%$ eran separados o divorciados, y 4\% eran viudos. En cuanto al nivel educativo, la mitad de los participantes no se había graduado del colegio, otro cuarto eran bachilleres, y el último cuatro tenía algunos estudios universitarios, de post-grado o una carrera técnica. En cuanto a filiación religiosa, un 67\% de los participantes eran Católicos, 20\% Evangélicos, y otro 13\% no revelaron su religión. Cincuenta y cinco por ciento de los participantes se identificaron como mestizo, 14\% afrocolombiano, 10\% indígena, y el resto eligió otra raza o etnicidad o más de un grupo.

Los participantes fueron seleccionados en una aproximación de un muestreo aleatorio para representar un rango amplio de sectores y perspectivas 
que incluyen: afrocolombianos, artesanos, campesinos, desmovilizados, desplazados, iglesias, indígenas, jóvenes, maestros, mujeres, pescadores, y víctimas. Se adoptó este proceso de reclutamiento por consideraciones de seguridad del equipo y los participantes, y para establecer más confianza en un tiempo corto. Visitas previas o colaboración con los contactos locales abrió la posibilidad de aplicar la encuesta con los sectores mencionados arriba.

\section{Instrumentos}

La encuesta se construyó a partir de cuestionarios para cada uno de los conceptos importantes del pilotaje y tuvo una duración de 45 minutos. La encuesta incluyó tanto instrumentos establecidos en psicología, como instrumentos más comunes en ciencias políticas y sociología, además de cuestionarios ya utilizados en la región. Algunos de los instrumentos ya estaban disponibles en español, pero para los casos donde sólo hay versiones en inglés disponibles, la autora realizó la traducción al español. El idioma fue corregido de una experta en inglés y español de Colombia independiente de este pilotaje. Después de estas redacciones, los miembros de las dos universidades locales, incluyendo profesores en psicología y trabajo social, revisaron los instrumentos para refinar el lenguaje y la redacción para que fuera comprensible y relevante para el contexto costeño. El equipo de investigación, incluyendo la autora y los quince investigadores de campo, discutieron las preguntas para sugerir que todos entendían los conceptos.

Experiencias con violencia. Para investigar los diferentes tipos e intensidades de la violencia, los participantes respondieron un instrumento de diez preguntas adaptado de Backer, Kulkarni, and Weinstein (2007). Indicaron no(=0) o sí(=1), si ellos mismos, o un miembro cercano de su familia, habían sufrido alguna de una lista de violaciones que incluyeron: detenido, arrestado o encarcelado sin causa; tortura física; tortura psicológica; golpeado; abuso, ataque o violación sexual; robo o extorsión; testigo de un asesinato o muerte de otra persona; amenazado con violencia; desplazado; y propiedad o casa despojada, dañada o destruida. Con el fin de capturar la experiencia con violencia fatal, los participantes indicaron si habían perdido alguien en su familia como resultado del conflicto. Y, por último, eligieron $s i ́(=1)$ o no $(=0)$ si estaban dispuestos perdonar a quién les hicieron daño a ellos mismos o a su familias durante el conflicto.

Salud Mental. Dos aspectos de la salud mental, bienestar y depresión, fueron incluidos en este pilotaje. Primero, el bienestar fue medido con el General Health 
Questionnaire (GHQ; Goldberg \& Williams, 1988), un instrumento de doce preguntas en una escala de cuatro puntos $(0=$ mucho menos que lo normal, a $3=$ mejor que lo normal). Los participantes señalaron la opción que mejor describe sus experiencias recientes frente a varias preguntas que incluyeron: ha sido capaz de concentrarse en lo que está haciendo, se ha sentido capaz de tomar decisiones, y se ha sentido más o menos feliz. Segundo, los síntomas de depresión fue medido con veinte preguntas en una escala de cuatro puntos $(0=$ raramente o nunca, a $3=$ la mayor parte o todo el tiempo $)$ sobre la frecuencia con que los participantes han sentido las siguientes cosas en la última semana, por ejemplo, mi sueño era inquieto, pasé ratos llorando, y no tenía ganas de hacer nada (Radloff, 1977). La consistencia interna de los dos instrumentos fue adecuada (GHQ, Cronbach's $\alpha=.76$; CESD, Cronbach's $\alpha=.87$ ).

Enfrentarse y adaptación. ${ }^{2}$ Los participantes respondieron a 24 preguntas que exploraban cómo se enfrentaron y adaptaron (cope with) a una situación difícil que tuvieron que enfrentar durante el último año (Perczek, Carver, Price, \& Pozo-Kaderman, 2000). Este instrumento es la versión breve que incluye dos preguntas para cada uno de doce subconceptos que representan diferentes maneras de enfrentar circunstancias difíciles: distraerse, tomar acciones concretas, negar, usar drogas o alcohol, apoyo emocional, desempeñar, hablar de lo malo, buscar lo positivo, planear, hacer bromas, aceptar, y buscar la religión. Habían cuatro opciones ( $1=$ no hice esto en lo absoluto, a $4=$ hice esto con mucha frecuencia), y ejemplos de unas de las preguntas incluyeron: recibí apoyo y comprensión de alguien, busqué algo positivo de la situación por la que estaba pasando, hice bromas acerca de esto, y recé o medité. La consistencia interna para todos estos subconceptos fue Cronbach's $\alpha=.74$.

Participación comunitaria. Para medir el nivel de participación comunitaria, los participantes indicaron si era un miembro activo $(=2)$, $\operatorname{inactivo}(=1)$, o no pertenecía $(=0)$ a los siguientes tipos de organizaciones: iglesia $u$ organización religiosa, deporte u organización recreativa, organización de arte o música, sindicato, grupo o partido político legal, organización humanitaria o caritativa, grupo académico, cooperativo, grupo de desarrollo comunitario, grupo juvenil, y Junta de Acción Comunal (JAC). La lista fue adaptada del World Social Values Survey (2005) y fue consultado con miembros de las universidades en la costa Caribe. Adicionalmente, los participantes tenían la opción para indicar otros grupos que no estaban en la lista. Cronbach's $\alpha=.75$ para este instrumento.

${ }^{2}$ Los autores del instrumento tradujeron "some ways of coping with difficult situations" como "algunas maneras de enfrentarse y adaptarse a situaciones difíciles." La escala en ingés y español es accesible ahgés y www.psy.miami.edu/faculty/ccarver/sclspan.html 
Confianza social. El instrumento para medir confianza social también fue adaptado del World Social Values Survey (2005). Confianza social se puede entender como un sentido que una persona tiene hacia diferentes grupos u organizaciones que implica una perspectiva positiva y la expectativa que el grupo referente no se va a violar las vulnerabilidades de uno; confianza es importante para cooperación y buenas relaciones (Tam, Hewstone, Kenworthy, \& Cairns, 2009). Los participantes respondieron en cuatro opciones $(0=$ no confió, a 3 =confió totalmente) para describir la profundidad de su confianza en personas de la familia, el barrio, que Ud. conoce, que conoce por primera vez, de otra religión, y de otra región. Para estas seis preguntas, la consistencia interna fue $\alpha=.72$.

Actitudes hacía la justica transicional. Para entender varias actitudes hacia diferentes dimensiones de justicia transicional, habían 23 preguntas sobre temas de verdad, reparación, y amnistía, (adaptadas de Backer, Kulkarni \& Weinstein, 2007). Un ejemplo de una pregunta sobre la verdad, pidió a los participantes que eligieran entre cinco opciones $(\mathrm{O}=$ muy desacuerdo, a $4=$ muy de acuerdo) si el pasado es algo que debería ser olvidado. Para examinar pensamientos sobre reparación, participantes indicaron si no tenía ninguna importancia $(=0)$ a era muy importante $(=3)$ que se haga algo para reparar las pérdidas que las personas sufrieron durante el conflicto. Les preguntamos a los participantes si estaban de acuerdo o en desacuerdo con la rebaja de penas dentro de la Ley de Justicia y Paz, y abajo, cuales condiciones aceptaría la amnistía es un resultado justo. Las opciones $(\mathrm{O}=$ muy desacuerdo, $4=$ muy de acuerdo $)$ incluyeron si los responsables dicen la verdad, reconocen el daño, piden perdón, muestran remordimiento, y reparan a las víctimas; adicionalmente, los participantes tenían la opción para elegir bajo ninguna circunstancia la amnistía es un resultado justo. Por último, los participantes respondieron sí( $=1)$ o no $(=0)$ si estaban dispuesto a perdonar a las personas que en medio del conflicto le causaron daño a Ud. o a su comunidad.

Democracia. La encuesta incluyó varias preguntas sobre actitudes y comportamientos en la democracia nacional y local (World Social Values Survey, 2005). Entre cuatro opciones de insatisfecho(=0) a muy satisfecho(=3), los participantes evaluaron la manera en que el gobierno nacional está manejando los asuntos del país. También eligieron entre dos opciones, si este país esta manejado por pocos intereses particulares o por toda la gente. Comparando democracia frente a otros tipos de gobierno, los participantes respondieron entre cuatro opciones si un sistema democrático es en general muy malo $(=0)$ a 
muy bueno(=3). Para entender las relaciones con las instituciones del estado local, otra pregunta pidió que los participantes señalaran a cuáles instituciones recurrirían si ellos o su comunidad tuvieran algún problema o necesidad. Cinco de los siete opciones fueron parte del gobierno local (alcalde o miembro de su gabinete, defensor del pueblo, fuerza pública, personero, e institución pública, por ejemplo Casa de Justicia, comisaría), mientras dos fueron parte de la sociedad civil (organización social e iglesia).

\section{RESULTADOS}

Las siguientes estadísticas fueron calculadas para explorar cómo las experiencias de violencia política afectan a las personas a nivel personal, específicamente en su salud mental, sus acciones y participación en la sociedad civil, y sus actitudes hacia la justica transicional y democracia.

\section{Experiencias con violencia}

Tres aspectos de violencia fueron incluidos en el pilotaje: experiencia personal (directa), violencia contra un miembro de su familia (indirecta), y muerte de un familiar durante el conflicto (fatal). Alrededor de 33\% de los participantes han sido afectado directamente (Ej., desplazado, golpeado, tortura física o mental), mientras un $64 \%$ ha sufrido estos tipos de violencia en su familia, y un 37\% ha perdido alguien en su familia. Se encontró una asociación significativa entre las personas que han enfrentado violencia directa y familiar $(r=.42, p<.01)$ y entre violencia fatal y no fatal contra la familia $(r=.44$, $p<.01)$. En resumen, personas que han experimentado violencia no fatal son miembros de familias también afectadas, e individuos en familias que han vivido violencia no fatal también han sido afectados por la muerte a un miembro de esta familia. Aunque es más probable que los que se identifican como víctimas son los que han sufrido algún tipo de violencia, en total 61\% de los participantes señala que se siente víctimas del conflicto.

\section{Impacto de la violencia en la salud mental}

Utilizando la variable control de género, el artículo analizó si la experiencia de los tres tipos de violencia predicen: peor bienestar y más altos niveles en los síntomas de depresión. Una regresión múltiple muestra que al control por medios estadísticos violencia contra la familia y violencia fatal, sus formas directas a la persona predijeron peor bienestar $(\beta=.19, p<.05)$, y este 
modelo completo explicó $14 \%$ de la variabilidad en bienestar $(\mathrm{F}(4,154)=6.06$, $p<.001)$. De la misma manera, violencia directa $(\beta=.19, p<.05)$ y violencia contra la familia $(\beta=.21, p<.05)$ predijeron más síntomas de depresión, controlado por la otra experiencia y género $(\mathrm{F}(4,145)=8.89, p<.001)$. Este modelo explicó $20 \%$ de la variabilidad de los síntomas de depresión. La muerte de un familiar no tenía una relación significativa a estas dimensiones de salud mental.

\section{Enfrentarse y adaptación}

Considerando el impacto negativo de la violencia en la salud mental, esta pregunta explora cómo los habitantes de la costa Caribe en general se enfrentan al estrés. La figura 2 muestra que hay mucha variabilidad en las dimensiones de enfrentarse al estrés que los costeños están utilizando para responder un periodo difícil en sus vidas.

Posteriormente se examinó las relaciones entre traumas específicos, salud mental, y diferentes tipos de enfrentarse. La idea fue identificar cuales tipos (generalmente positivos, neutrales, o negativos) de enfrentarse y adaptar están utilizando los individuos y después se analizará si son efectivos en la protección de la salud mental. El cuadro 1 contiene los patrones de las correlaciones de interés entre dos aspectos de salud mental (bienestar y depresión), dos tipos de violencia (directa y familiar), y cinco dimensiones de enfrentarse a situaciones difíciles. Este rango muestra diferentes aspectos de adaptación típicamente considerado positivos (apoyo emocional y cambiar a una percepción positiva), neutrales (religión), y negativos (uso de alcohol o droga, y comportamiento para evitar los problemas). En general, recibir apoyo y comprensión emocional disminuye problemas de salud mental $(r=-.20, p<.01 ; r=-.30, p<.01)$, mientras los comportamientos de evitar los problemas están conectados a peor salud mental $(r=.20, p<.05 ; r=-.29$, $p<.01)$ y el uso de alcohol disminuye la salud mental $(r=.19, p<.05)$. En adición, la habilidad de cambiar a una perspectiva positiva cuando se enfrentan situaciones difíciles baja la depresión $(r=-.19, p<.05)$. Es importante notar que personas que han sufridos altos niveles de violencia (directa y familiar) utilizan menos apoyo emocional $(r=.16, p<.05 ; r=-.23, p<.01)$. Repasamos las implicaciones de este conjunto de resultados en la discusión, pero notamos ahora que estos patrones indicarían la necesidad por crear intervenciones que aumenten el apoyo emocional para los afectados por la violencia. 
Figura 2: Estilos de enfrentarse y adaptar a situaciones difíciles (Promedios, rango 2 a 8)

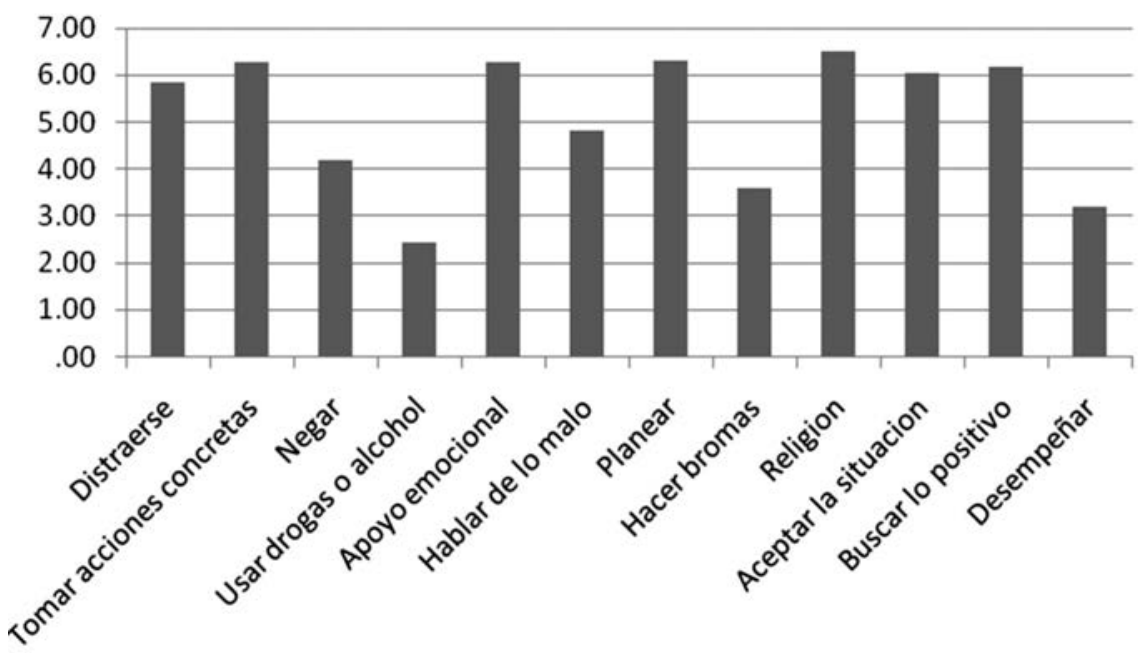

Cuadro 1: Correlaciones entre salud mental, violencia, y estilos de Enfrentarse

\begin{tabular}{|l|c|c|c|c|c|c|}
\hline & $\begin{array}{c}\text { Apoyo } \\
\text { emocio- } \\
\text { nal }\end{array}$ & $\begin{array}{c}\text { Verlo } \\
\text { positivo }\end{array}$ & $\begin{array}{c}\text { Uso de } \\
\text { alcohol }\end{array}$ & $\begin{array}{c}\text { Evitar los } \\
\text { problemas }\end{array}$ & $\begin{array}{c}\text { Violencia } \\
\text { individual }\end{array}$ & $\begin{array}{c}\text { Violencia a } \\
\text { un miembro } \\
\text { de su familia }\end{array}$ \\
\hline Bienestar & + & + & - & - & - & $n s$ \\
\hline Depresión & + & $n s$ & $n s$ & - & $n s$ & - \\
\hline Violencia individual & - & $n s$ & $n s$ & $n s$ & - & + \\
\hline $\begin{array}{l}\text { Violencia a un miem- } \\
\text { bro de su familia }\end{array}$ & - & $n s$ & $n s$ & $n s$ & + & - \\
\hline
\end{tabular}

Nota: $+=$ positivo, $-=$ negativo, $n s=$ no significativo

\section{Participación y confianza social}

En el marco teórico la participación en las organizaciones de la comunidad y confianza social entre personas fueron dos elementos importantes. Controlando la variable género y otras experiencias de violencia, la experiencia de violencia directa predijo mayor participación $(\beta=.20, p<.05)$. Este modelo completo explicó $15 \%$ de la variabilidad de participación activa $(\mathrm{F}(4,158)=7.12, p<.001)$. Estos resultados nos plantean la pregunta: ¿A qué tipo de organizaciones pertenecen las personas que han sido afectadas directamente por la violencia política? Las correlaciones a esta pregunta revelan que las personas que han sufrido violencia directa durante el conflicto son más activos en deportes u organizaciones recreativas $(r=.16, p<.05)$, sindicatos $(r=.36, p<.01)$, grupos o partidos políticos legales $(r=.16, p<.05)$, grupos académicos $(r=.23, p<.01)$, y grupos de desarrollo comunitario $(r=.18, p<.05)$. 


\section{Actitudes hacía la justica transicional, amnistía, y perdón}

Consideramos varios mecanismos de la justicia transicional, y en general los participantes le daban mucha importancia a varios de estos mecanismos. Un análisis de los componentes principales (PCA) reveló tres fenómenos distintos: (1) responsabilidad de los victimarios (Ej., que los responsables confronten las consecuencias por lo que han hecho), (2) reparación y cambios estructurales (Ej., que las circunstancias que contribuyeron al conflicto cambien para bien), y (3) reconocimiento público de las víctimas y los daños causados (Ej., que los hechos sobre lo que sucedió sean investigados y llevados a conocimiento público) (Cronbach's $\alpha=.71, .70, .64$, respectivamente). Es posible que el rango de las respuestas fuera limitado por un "efecto del techo"; es decir, ningún participante respondió bajo un promedio de desacuerdo a los diferentes mecanismos y más de $81 \%$ estaban muy de acuerdo con la aplicación de estos mecanismos.

Del mismo modo, la mayoría de los participantes estaban muy de acuerdo con la amnistía condicional, o sea con la presencia de otros mecanismos de justicia transicional, por ejemplo, si los responsables por las violaciones confiesan la verdad sobre lo que han hecho. Menos del 35\% estaban en desacuerdo con la amnistía condicional, mientras aproximadamente 25\% estaban muy de acuerdo en ciertas condiciones. Sin embargo, 70\% de los participantes estaban en desacuerdo con la Ley de Justicia y Paz. Parece que aunque los participantes reconocían que amnistía legal puede ser parte del proceso para transformar el conflicto, la mayor parte no creían que la Ley de Justicia y Paz cumplió con estas condiciones.

Figura 5: Actitudes hacia el perdón y la Ley de Justicia y Paz

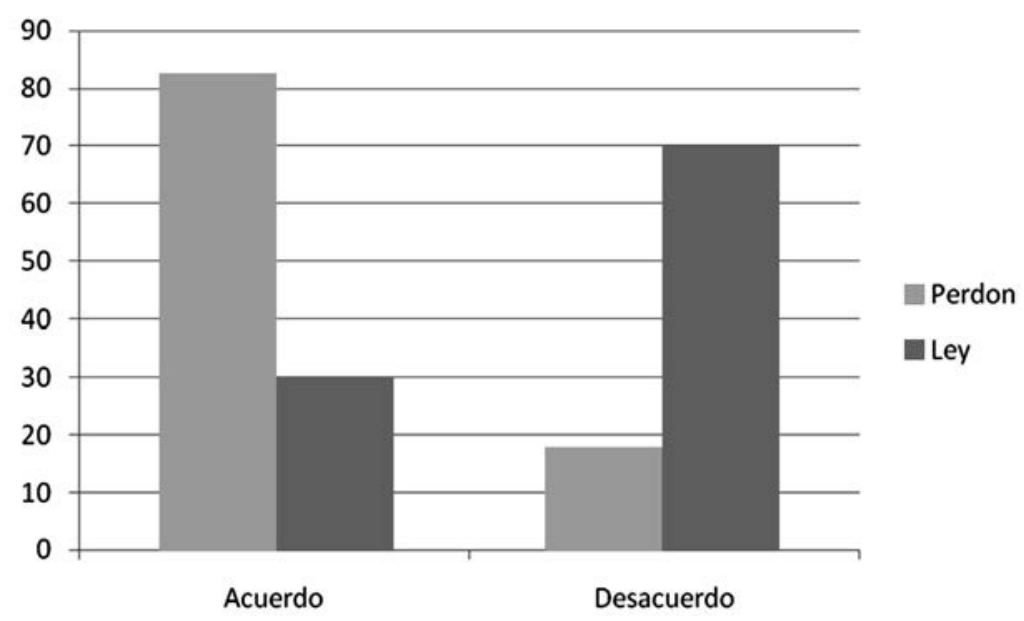


Con respecto al perdón, aunque este caso no llegó exactamente a un nivel significativo $(\mathrm{t}(145)=1.95$, variables iguales no presumidos, $p=.053)$, un resultado interesante es que quienes han sufrido más violencia directa en promedio son más abiertos a perdonar. Adicionalmente, los participantes que señalaron que estaban más inclinados a perdonar a los que les hicieron daño durante el conflicto, también querían que los responsables presentaran disculpas a las víctimas $(\mathrm{t}(154)=2.57, p<.05)$. Al final, $82 \%$ de los participantes confirmaron que estaban dispuestos a perdonar a las personas que en medio del conflicto les causaron daño a ellos y a su comunidad.

\section{Democracia}

Los datos descriptivos sobre las percepciones de los participantes hacia la democracia en Colombia son variados. Aunque el 79\% dijo que la democracia es un sistema de gobernanza muy buena y otro $21 \%$ dijo que es bastante buena para este país (Figura 3), casi la mitad (46\%) dijo que no estaba satisfecho con la manera que el gobierno nacional está manejando los asuntos del país (Figura 4). De la misma manera, 60\% dijo que pensaba que el país estaba manejado con intereses particulares que solo se preocupan por sí mismos, y por el contrario, 40\% dijo que el país estaba manejado para el beneficio de todas las personas. A nivel local, el promedio de los participantes reflejó que si ellos o su comunidad tuvieran algún problema o necesidad hay $3.38(S D=2.39)$ instituciones, de una lista de siete, a nivel local a las que pueden recurrir.

Figura 3: Perspectiva de la democracia en general

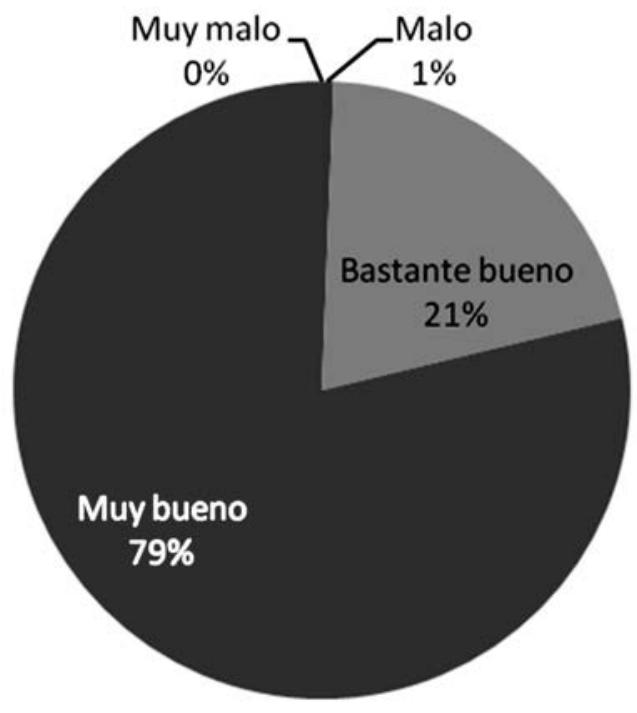

Figura 4: Satisfacción del Gobierno Nacional

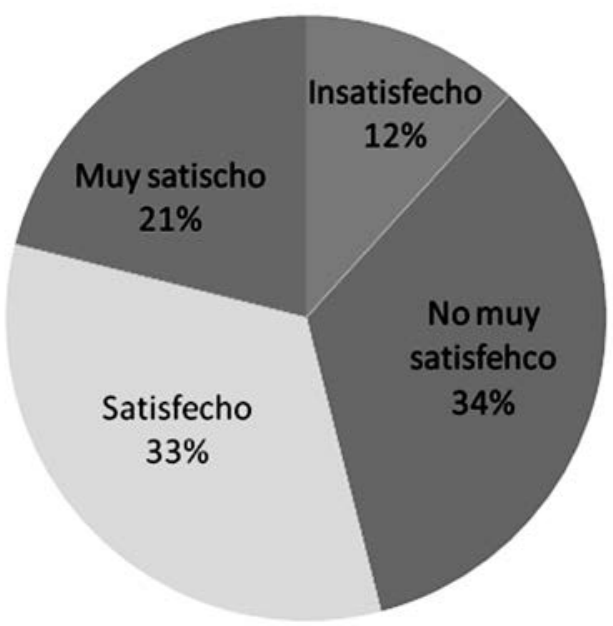




\section{DISCUSIÓN E IMPLICACIONES}

En este contexto, la costa Caribe colombiana constituye un caso importante para estudiar las relaciones entre violencia, salud mental, participación y actitudes hacia la justicia transicional, mientras los individuos viven dentro de una realidad violenta. En el futuro, estos resultados serán comparados con las entrevistas y el grupo focal para entender procesos más colectivos. Esperando estas comparaciones, hay unos patrones importantes para resaltar del análisis cuantitativo. Parecido a otros contextos de conflicto, la violencia política directa e indirecta tiene un impacto negativo en la salud mental. Sin embargo hay evidencia de resiliencia, que se puede definir como resistencia mental cuando confrontan situaciones difíciles y la habilidad de mantener un sentido de equilibrio estable (Bonanno, 2004). Por ejemplo, personas que reciben más apoyo emocional presentan menos problemas en su salud mental; sin embargo, las personas que han sufrido mayores grados de violencia tienden a recurrir menos a este recurso interpersonal.

En respuesta a cómo el conflicto ha afectado la participación, se percibe que la violencia directa motiva a las personas a participar en organizaciones en sus comunidades. Considerando que tenemos datos correspondientes únicamente a un periodo de tiempo, no podemos desenredar la dirección de esta relación en este artículo; por ejemplo, si es posible que las personas más activas en la comunidad tuvieran un mayor riesgo de ser víctima de violencia. Sin embargo, las preguntas de la experiencia de violencia tiene que ver con el pasado y las preguntas de participación tiene que ver con los grupos actuales. Además, no encontramos evidencia que la violencia esté relacionada con la confianza social. Aunque no deberíamos interpretar estadísticas no significativas, este vacío llama la atención para más investigación y reformulación de los instrumentos y/o teoría. Parece que los habitantes de la costa Caribe, en promedio, pueden mantener un nivel de confianza total con sus familias y confianza más o menos en sus barrios y con personas conocidas a pesar del conflicto prolongado. Además las cifras de democracia indican que aunque la mitad no estaba satisfecha con la gobernanza nacional, a nivel de la comunidad había alguna confianza en las instituciones del gobierno local.

Con respecto a las actitudes hacia los mecanismos de justicia transicional, los resultados son complejos. En general los participantes, incluyendo las víctimas, expresaron gran afinidad a la posibilidad de perdón y aprobaron la amnistía siempre y cuando los responsables digan la verdad, reconozcan el 
daño que causaron, presenten disculpas, muestren remordimiento, y reparen a las víctimas. La Ley de Justicia y Paz contempla muchas de estas condiciones en su mandato, sin embargo la incapacidad de la ley para cumplirlas ha traído como consecuencia que los participantes no apoyan esta iniciativa. Considerando la falta de implementación de las condiciones que están en la Ley de Justicia y Paz, los participantes manifiestan su desacuerdo con la misma.

De estos resultados, salieron las siguientes recomendaciones:

Implicación \# 1: Hay diferente tipos de víctimas de violencia, y los programas necesitan tener este factor en cuenta;

Implicación \#2: Crear programas para las víctimas dirigidos a aumentar el apoyo emocional y la posibilidad de buscar algo bueno en lo que estaba pasando; Implicación \#3: Establecer y fortalecer organizaciones comunitarias para fomentar oportunidades de participación;

Implicación \# 4: Trabajar y crear intervenciones a nivel local donde exista mayor confianza y conocimiento;

Implicación \# 5: Respetar y cumplir con las condiciones de justicia transicional para mantener el apoyo de la base.

Los resultados de este artículo tienen implicaciones para una audiencia con interés en la micro-dinámica de conflicto y cómo individuos y grupos deciden participar y contribuir en un sistema político. Igualmente, se puede incluir a miembros de la sociedad civil, ONGs, entidades del Estado tanto a nivel local como regional, y practicantes y académicos interesados en la transformación de conflictos y justicia transicional.

La esperanza es que esta investigación no solo haya sido un ejercicio intelectual y que estos datos y recomendaciones no solo queden en el papel. Por el contrario, esperamos que líderes y grupos comunitarios, o comunidades semejantes, puedan usar esta información empírica en la construcción de programas y en futuras intervenciones. Los vínculos entre la violencia, la salud mental, la participación, y las percepciones de los mecanismos de justicia transicional tienen implicaciones para la posibilidad de construir una paz verdadera en la costa Caribe colombiana. Otros países pueden aprender de las experiencias, retos, y logros enfrentados a nivel individual, comunitario, regional, y nacional con respecto a estos temas dentro de un contexto de conflicto prolongado.

PalObra No. 12. Agosto de 2010 - Julio de 2011 


\section{BIBLIOGRAFIA}

Backer, D., Kulkarni, A., \& Weinstein, H. (2007). West Africa transitional justice project - Liberia Questionnaire (First Wave).

Bonnano, G.A. (2004). Loss, trauma, and human resilience: Have we underestimated the human capacity to thrive after extremely adverse events? American Psychologist, 59(20, 20-28.

Bouvier, V. (Ed.) (2009). Colombia: Building peace in a time of war. Washington DC: United States Institute of Peace.

Bronfenbrenner, U. (1979). The ecology of human development: Experiments by nature and design. Cambridge, MA: Harvard University Press.

Goldberg, D. P., \& Williams, P. (1988). A user's guide to the general health questionnaire. Windsor, United Kingdom: NFER-Nelson.

Grodsky, Brian (2009) Re-Ordering Justice: Towards a New Methodological Approach to Studying Transitional Justice. Journal of Peace Research 46(6): 819-837.

Hamber, B. (2009). Transforming societies after political violence: Truth, reconciliation, and mental health. New York: Springer Series.

International Center for Transitional Justice (ICTJ) (2008). Panorama cualitativo y cuantitivo nacional, con énfasis en cuatro regiones. Retrieved from http://www.ictjcolombia.org/edicion07/ docs/Libro\%20final\%20Panoramas.pdf

International Center for Transitional Justice (ICTJ) (2011). What is Transitional Justice? Retrieved from http://ictj.org/about/transitional-justice

Lederach, J.P. (1997) Building peace: Sustainable reconciliation in divided societies. Washington, DC: United States Institute of Peace.

Lederach, J.P. (2005). Moral imagination: The art and soul of building peace. New York: Oxford University Press.

Martín-Bar6, I. (1996). Writings for a liberation psychology. Cambridge: Harvard University Press.

Polity IV Country Report 2010: Colombia. Retrieved from http://www.systemicpeace.org/ polity/Colombia2010.pdf

Perczek, R., Carver, C. S., Price, A. A., \& Pozo-Kaderman, C. (2000). Coping, mood, and aspects of personality in Spanish translation and evidence of convergence with English versions. 
Journal of Personality Assessment, 74, 63-87. Retrieved from http://www.psy.miami.edu/ faculty/ccarver/sclspan.html

Radloff, L. S. (1977). The CES-D Scale: A self-report depression scale for research in the general population. Applied Psychological Measurement, 1, 385-401. Spanish CES-D Retrieved from http://www.psy.miami.edu/faculty/ccarver/sclspan.html

Staub, E., \& Vollhardt, J. (2008). Altruism born of suffering: The roots of caring and helping after victimization and other trauma. American Journal of Orthopsychiatry, 78(3), 267-280.

Shapiro, D.L. (2010). Relational identity theory: A systematic approach for transferring the emotional dimension of conflict. American Psychologist, 65, 634-645.

Tam, T., Hewstone, M., Kenworthy, J., \& Cairns, E. (2009). Intergroup trust in Northern Ireland. Personality and Social Psychology Bulletin. 35(1), 45-59.

World Social Values Survey (2005). Retrieved from http://www.worldvaluessurvey.org/wvs/ articles/folder_published/survey_2005. 